\title{
RELATIVE VALUE ITERATION FOR STOCHASTIC DIFFERENTIAL GAMES
}

\author{
ARI ARAPOSTATHIS, VIVEK S. BORKAR, AND K. SURESH KUMAR
}

\begin{abstract}
We study zero-sum stochastic differential games with player dynamics governed by a nondegenerate controlled diffusion process. Under the assumption of uniform stability, we establish the existence of a solution to the Isaac's equation for the ergodic game and characterize the optimal stationary strategies. The data is not assumed to be bounded, nor do we assume geometric ergodicity. Thus our results extend previous work in the literature. We also study a relative value iteration scheme that takes the form of a parabolic Isaac's equation. Under the hypothesis of geometric ergodicity we show that the relative value iteration converges to the elliptic Isaac's equation as time goes to infinity. We use these results to establish convergence of the relative value iteration for risk-sensitive control problems under an asymptotic flatness assumption.
\end{abstract}

\section{INTRODUCTION}

In this paper we consider a relative value iteration for zero-sum stochastic differential games. This relative value iteration is introduced in [1] for stochastic control, and we follow the method introduced in this paper.

In Section 2, we prove the existence of a solution to the Isaac's equation corresponding to the ergodic zero-sum stochastic differential game. We do not assume that the data or the running payoff function is bounded, nor do we assume geometric ergodicity, so our results extend the work in [5]. In Section 3, we introduce a relative value iteration scheme for the zero-sum stochastic differential game and prove its convergence under a hypothesis of geometric ergodicity. In Section 4, we apply the results from Section 3 and study a value iteration scheme for risk-sensitive control under an asymptotic flatness assumption.

\section{Problem Description}

We consider zero-sum stochastic differential games with state dynamics modeled by a controlled nondegenerate diffusion process $X=\{X(t): 0 \leq t<\infty\}$, and subject to a long-term average payoff criterion.

2.1. State dynamics. Let $U_{i}, i=1,2$, be compact metric spaces and $V_{i}=\mathcal{P}\left(U_{i}\right)$ denote the space of all probability measures on $U_{i}$ with Prohorov topology. Let

$$
\bar{b}: \mathbb{R}^{d} \times U_{1} \times U_{2} \rightarrow \mathbb{R}^{d} \quad \text { and } \quad \sigma: \mathbb{R}^{d} \rightarrow \mathbb{R}^{d \times d}
$$

1991 Mathematics Subject Classification. Primary, 93E15, 93E20; Secondary, 60J25, 60J60, 90C40.

Ari Arapostathis was supported in part by ONR under the Electric Ship Research and Development Consortium.

Vivek Borkar was supported in part by Grant \#11IRCCSG014 from IRCC, IIT, Mumbai. 
be measurable functions. Assumptions on $\bar{b}$ and $\sigma$ will be specified later. Define $b: \mathbb{R}^{d} \times$ $V_{1} \times V_{2} \rightarrow \mathbb{R}^{d}$ as

$$
b\left(x, v_{1}, v_{2}\right):=\int_{U_{1}} \int_{U_{2}} \bar{b}\left(x, u_{1}, u_{2}\right) v_{1}\left(\mathrm{~d} u_{1}\right) v_{2}\left(\mathrm{~d} u_{2}\right),
$$

for $x \in \mathbb{R}^{d}, v_{1} \in V_{1}$ and $v_{2} \in V_{2}$. We model the controlled diffusion process $X$ via the Itô s.d.e.

$$
\mathrm{d} X(t)=b\left(X(t), v_{1}(t), v_{2}(t)\right) \mathrm{d} t+\sigma(X(t)) \mathrm{d} W(t) .
$$

All processes on (2.1) are defined in a common probability space $(\Omega, \mathcal{F}, P)$ which is assumed to be complete. The process $W=\{W(t): 0 \leq t<\infty\}$ is an $\mathbb{R}^{d}$-valued standard Wiener process which is independent of the initial condition $X_{0}$ of (2.1). Player $i$, with $i=1,2$, controls the dynamics $X$ through her strategy $v_{i}(\cdot)$, a $V_{i}$-valued process which is jointly measurable in $(t, \omega) \in[0, \infty) \times \Omega$ and non-anticipative, i.e., for $s<t, W(t)-W(s)$ is independent of

$$
\mathcal{F}_{s}:=\text { the completion of } \sigma\left(X_{0}, v_{1}(r), v_{2}(r), W(r), r \leq s\right) .
$$

We denote the set of all such controls (admissible controls) for player $i$ by $\mathcal{U}_{i}, i=1,2$.

Assumptions on the Data: We assume the following conditions on the coefficients $\bar{b}$ and $\sigma$ to ensure existence of a unique solution to (2.1).

(A1) The functions $\bar{b}$ and $\sigma$ are locally Lipschitz continuous in $x \in \mathbb{R}^{d}$, uniformly over $\left(u_{1}, u_{2}\right) \in U_{1} \times U_{2}$, and have at most a linear growth rate in $x \in \mathbb{R}^{d}$, i.e., for some constant $\kappa$,

$$
\left\|\bar{b}\left(x, u_{1}, u_{2}\right)\right\|^{2}+\|\sigma(x)\|^{2} \leq \kappa\left(1+\|x\|^{2}\right) \quad \forall\left(x, u_{1}, u_{2}\right) \in \mathbb{R}^{d} \times U_{1} \times U_{2},
$$

where $\|\sigma\|^{2}:=\operatorname{trace}\left(\sigma \sigma^{\top}\right)$, with ${ }^{\top}$ denoting the transpose. Also $\bar{b}$ is continuous.

(A2) For each $R>0$ there exists a constant $\kappa(R)>0$ such that

$$
z^{\top} a(x) z \geq \kappa(R)\|z\|^{2} \quad \text { for all }\|x\| \leq R \text { and } z \in \mathbb{R}^{d},
$$

where $a:=\sigma \sigma^{\top}$.

Definition 2.1. For $f \in C^{2}\left(\mathbb{R}^{d}\right)$ define

$$
\bar{L} f\left(x, u_{1}, u_{2}\right):=\bar{b}\left(x, u_{1}, u_{2}\right) \cdot \nabla f(x)+\frac{1}{2} \operatorname{tr}\left(a(x) \nabla^{2} f(x)\right)
$$

for $x \in \mathbb{R}^{d}$ and $\left(u_{1}, u_{2}\right) \in U_{1} \times U_{2}$. Also define the relaxed extended controlled generator $L$ by

$$
L f\left(x, v_{1}, v_{2}\right):=\int_{U_{1}} \int_{U_{2}} L f\left(x, u_{1}, u_{2}\right) v_{1}\left(\mathrm{~d} u_{1}\right) v_{2}\left(\mathrm{~d} u_{2}\right), \quad f \in C^{2}\left(\mathbb{R}^{d}\right),
$$

for $x \in \mathbb{R}^{d}$ and $\left(v_{1}, v_{2}\right) \in V_{1} \times V_{2}$.

We denote the set of all stationary Markov strategies of player $i$ by $\mathcal{M}_{i}, i=1,2$. 
2.2. Zero-sum ergodic game. Let $\bar{h}: \mathbb{R}^{d} \times U_{1} \times U_{2} \rightarrow[0, \infty)$ be a continuous function, which is also locally Lipschitz continuous in its first argument. We define the relaxed running payoff function $h: \mathbb{R}^{d} \times V_{1} \times V_{2} \rightarrow[0, \infty)$ by

$$
h\left(x, v_{1}, v_{2}\right):=\int_{U_{1}} \int_{U_{2}} \bar{h}\left(x, u_{1}, u_{2}\right) v_{1}\left(\mathrm{~d} u_{1}\right) v_{2}\left(\mathrm{~d} u_{2}\right) .
$$

Player 1 seeks to maximize the average payoff given by

$$
\liminf _{T \rightarrow \infty} \frac{1}{T} E_{x}\left[\int_{0}^{T} h\left(X(t), v_{1}(t), v_{2}(t)\right) \mathrm{d} t\right]
$$

over all admissible controls $v_{1} \in \mathcal{U}_{1}$, while Player 2 seeks to minimize (2.2) over all $v_{2} \in$ $\mathcal{U}_{2}$. Here $E_{x}$ is the expectation operator corresponding to the probability measure on the canonical space of the process starting at $X(0)=x$.

Since we shall analyze the average payoff as a limiting case of the discounted payoff in the 'vanishing discount' limit, we shall also consider the infinite horizon discounted payoff

$$
E_{x}\left[\int_{0}^{\infty} \mathrm{e}^{-\alpha t} h\left(X(t), v_{1}(t), v_{2}(t)\right) \mathrm{d} t\right],
$$

where $\alpha>0$ is the discount factor.

Assumptions on Ergodicity: We consider the following ergodicity assumptions:

(A3) There exist a positive inf-compact function $\mathcal{V} \in C^{2}\left(\mathbb{R}^{d}\right)$ and positive constants $k_{0}$, $k_{1}$ and $k_{2}$ such that

$$
\begin{aligned}
\bar{L} \mathcal{V}\left(x, u_{1}, u_{2}\right) & \leq k_{0}-2 k_{1} \mathcal{V}(x), \\
\max _{u_{1} \in U_{1}, u_{2} \in U_{2}} \bar{h}\left(x, u_{1}, u_{2}\right) & \leq k_{2} \mathcal{V}(x)
\end{aligned}
$$

for all $\left(u_{1}, u_{2}\right) \in U_{1} \times U_{2}$, and $x \in \mathbb{R}^{d}$. Without loss of generality we assume $\mathcal{V} \geq 1$.

$\left(\mathrm{A} 3^{\prime}\right)$ There exist nonnegative inf-compact functions $\mathcal{V} \in C^{2}\left(\mathbb{R}^{d}\right)$ and $g \in C\left(\mathbb{R}^{d}\right)$, and positive constants $k_{0}$ and $k_{2}$ such that

$$
\begin{aligned}
\bar{L} \mathcal{V}\left(x, u_{1}, u_{2}\right) & \leq k_{0}-g(x), \\
\max _{u_{1} \in U_{1}, u_{2} \in U_{2}} \bar{h}\left(x, u_{1}, u_{2}\right) & \leq k_{2} g(x)
\end{aligned}
$$

for all $\left(u_{1}, u_{2}\right) \in U_{1} \times U_{2}$, and $x \in \mathbb{R}^{d}$. Also,

$$
\frac{\max _{u_{1} \in U_{1}, u_{2} \in U_{2}} \bar{h}\left(x, u_{1}, u_{2}\right)}{g(x)} \underset{\|x\| \rightarrow \infty}{\longrightarrow} 0 .
$$

Without loss of generality we assume $\mathcal{V} \geq 1$ and $g \geq 1$.

In this section we use assumption $\left(\mathrm{A} 3^{\prime}\right)$, while in Section 3 we employ (A3) which is stronger and equivalent to geometric ergodicity in the time-homogeneous Markov case.

For the uncontrolled (i.e., Markov) case, $\left(\mathrm{A} 3^{\prime}\right)$ is the so called 'g-norm ergodicity' in the terminology of [11] which implies, in addition to convergence of laws to a unique stationary distribution, convergence of $\frac{1}{t} \int_{0}^{t} E[f(X(s))] \mathrm{d} s$ to the corresponding stationary expectation as $t \uparrow \infty$ for all $f$ with growth rate at most that of $g$ and vice versa. Assumption (A3) corresponds to the same with $h=V$ and implies in particular exponential convergence 
to stationary averages (and vice versa). This is the so called geometric ergodicity. When $\left(\mathrm{A} 3^{\prime}\right)$ holds in the controlled case, it implies in particular tightness of stationary distributions attainable under stationary Markov controls. In fact this condition is necessary and sufficient. See [2, Lemma 3.3.4] for this and other equivalent characterizations. Thus (A3') is the best possible condition for uniform stability in this sense. While the results of [1] can be extended to control problems when instability is possible but is penalized by the cost structure, this does not extend naturally to the zero sum game, because what is penalty for one agent is a reward for the other.

We start with a theorem which characterizes the value of the game under a discounted infinite horizon criterion. For this we need the following notation: For a continuous function $\mathcal{V}: \mathbb{R}^{d} \rightarrow(0, \infty), C_{\mathcal{V}}\left(\mathbb{R}^{d}\right)$ denotes the space of functions in $C\left(\mathbb{R}^{d}\right)$ satisfying $\sup _{x \in \mathbb{R}^{d}}\left|\frac{f(x)}{\mathcal{V}(x)}\right|<$ $\infty$. This is a Banach space under the norm

$$
\|f\|_{\mathcal{V}}:=\sup _{x \in \mathbb{R}^{d}}\left|\frac{f(x)}{\mathcal{V}(x)}\right| .
$$

Theorem 2.1. Assume (A1), (A2) and (A3'). For $\alpha>0$, there exists a solution $\varphi_{\alpha} \in$ $C_{\mathcal{V}}\left(\mathbb{R}^{d}\right) \cap C^{2}\left(\mathbb{R}^{d}\right)$ to the p.d.e.

$$
\begin{aligned}
\alpha \psi_{\alpha}(x) & =\min _{v_{2} \in V_{2}} \max _{v_{1} \in V_{1}}\left[L \psi_{\alpha}\left(x, v_{1}, v_{2}\right)+h\left(x, v_{1}, v_{2}\right)\right] \\
& =\max _{v_{1} \in V_{1}} \min _{v_{2} \in V_{2}}\left[L \psi_{\alpha}\left(x, v_{1}, v_{2}\right)+h\left(x, v_{1}, v_{2}\right)\right]
\end{aligned}
$$

and is characterized by

$$
\begin{aligned}
\psi_{\alpha}(x) & =\sup _{v_{1} \in \mathcal{U}_{1}} \inf _{v_{2} \in \mathcal{U}_{2}} \mathrm{E}_{x}\left[\int_{0}^{\infty} \mathrm{e}^{-\alpha t} h\left(X(t), v_{1}(t), v_{2}(t)\right) \mathrm{d} t\right] \\
& =\inf _{v_{2} \in \mathcal{U}_{2}} \sup _{v_{1} \in \mathcal{U}_{1}} \mathrm{E}_{x}\left[\int_{0}^{\infty} \mathrm{e}^{-\alpha t} h\left(X(t), v_{1}(t), v_{2}(t)\right) \mathrm{d} t\right] .
\end{aligned}
$$

Proof. Let $B_{R}$ denote the open ball of radius $R$ centered at the origin in $\mathbb{R}^{d}$. The p.d.e.

$$
\begin{aligned}
\alpha \varphi_{\alpha}^{R}(x) & =\min _{v_{2} \in V_{2}} \max _{v_{1} \in V_{1}}\left[L \varphi_{\alpha}^{R}\left(x, v_{1}, v_{2}\right)+h\left(x, v_{1}, v_{2}\right)\right], \\
\varphi_{\alpha}^{R} & =0 \quad \text { on } \partial B_{R}
\end{aligned}
$$

has a unique solution $\varphi_{\alpha}^{R}$ in $C^{2}\left(B_{R}\right) \cap C\left(\overline{B_{R}}\right)$, see [8, Theorem 15.12, p. 382]. Since

$$
\min _{v_{2} \in V_{2}} \max _{v_{1} \in V_{1}}\left[L \varphi_{\alpha}^{R}\left(x, v_{1}, v_{2}\right)+h\left(x, v_{1}, v_{2}\right)\right]=\max _{v_{1} \in V_{1}} \min _{v_{2} \in V_{2}}\left[L \varphi_{\alpha}^{R}\left(x, v_{1}, v_{2}\right)+h\left(x, v_{1}, v_{2}\right)\right],
$$

it follows that $\varphi_{\alpha}^{R} \in C^{2}\left(B_{R}\right) \cap C\left(\overline{B_{R}}\right)$ is also a solution to

$$
\begin{aligned}
\alpha \varphi_{\alpha}^{R}(x) & =\max _{v_{1} \in V_{1}} \min _{v_{2} \in V_{2}}\left[L \varphi_{\alpha}^{R}\left(x, v_{1}, v_{2}\right)+h\left(x, v_{1}, v_{2}\right)\right], \\
\varphi_{\alpha}^{R} & =0 \quad \text { on } \partial B_{R} .
\end{aligned}
$$


Let $v_{1 \alpha}^{R}: B_{R} \rightarrow V_{1}$ be a measurable selector for the maximizer in (2.5) and $v_{2 \alpha}^{R}: B_{R} \rightarrow V_{2}$ be a measurable selector for the minimizer in (2.4). If we let

$$
F\left(x, v_{1} ; \varphi_{\alpha}^{R}\right):=\min _{v_{2} \in V_{2}}\left[L \varphi_{\alpha}^{R}\left(x, v_{1}, v_{2}\right)+h\left(x, v_{1}, v_{2}\right)\right],
$$

then $\left(x, v_{1}\right) \mapsto F\left(x, v_{1} ; \varphi_{\alpha}^{R}\right)$ is continuous and also Lipschitz in $x$, and $\varphi_{\alpha}^{R}$ satisfies

$$
\begin{aligned}
\alpha \varphi_{\alpha}^{R}(x) & =F\left(x, v_{1 \alpha}^{R}(x) ; \varphi_{\alpha}^{R}\right) \\
& =\min _{v_{2} \in V_{2}}\left[L \varphi_{\alpha}^{R}\left(x, v_{1 \alpha}^{R}(x), v_{2}\right)+h\left(x, v_{1 \alpha}^{R}(x), v_{2}\right)\right], \\
\varphi_{\alpha}^{R} & =0 \quad \text { on } \partial B_{R} .
\end{aligned}
$$

By a routine application of Itô's formula, it follows that

$$
\varphi_{\alpha}^{R}(x)=\inf _{v_{2} \in \mathcal{U}_{2}} \mathrm{E}_{x}\left[\int_{0}^{\tau_{R}} \mathrm{e}^{-\alpha t} h\left(X(t), v_{1 \alpha}^{R}(X(t)), v_{2}(t)\right) \mathrm{d} t\right]
$$

where

$$
\tau_{R}:=\inf \{t \geq 0:\|X(t)\| \geq R\}
$$

and $X$ is the solution to (2.1) corresponding to the control pair $\left(v_{1 \alpha}^{R}, v_{2}\right)$, with $v_{2} \in \mathcal{U}_{2}$.

Repeating the above argument with the outer minimizer $v_{2 \alpha}^{R}$ of $(2.4)$, we similarly obtain

$$
\varphi_{\alpha}^{R}(x)=\sup _{v_{1} \in \mathcal{U}_{1}} \mathrm{E}_{x}\left[\int_{0}^{\tau_{R}} \mathrm{e}^{-\alpha t} h\left(X(t), v_{1}(t), v_{2 \alpha}^{R}(X(t))\right) \mathrm{d} t\right] .
$$

Combining (2.6) and (2.7), we obtain

$$
\begin{aligned}
\inf _{v_{2} \in \mathcal{U}_{2}} \sup _{v_{1} \in \mathcal{U}_{1}} \mathrm{E}_{x}\left[\int_{0}^{\tau_{R}} \mathrm{e}^{-\alpha t} h(X(t),\right. & \left.\left.v_{1}(t), v_{2}(t)\right) \mathrm{d} t\right] \leq \varphi_{\alpha}^{R}(x) \\
\leq & \sup _{v_{1} \in \mathcal{U}_{1}} \inf _{v_{2} \in \mathcal{U}_{2}} \mathrm{E}_{x}\left[\int_{0}^{\tau_{R}} \mathrm{e}^{-\alpha t} h\left(X(t), v_{1}(t), v_{2}(t)\right) \mathrm{d} t\right],
\end{aligned}
$$

which implies that

$$
\begin{aligned}
\varphi_{\alpha}^{R}(x) & =\sup _{v_{1} \in \mathcal{U}_{1}} \inf _{v_{2} \in \mathcal{U}_{2}} \mathrm{E}_{x}\left[\int_{0}^{\tau_{R}} \mathrm{e}^{-\alpha t} h\left(X(t), v_{1}(t), v_{2}(t)\right) \mathrm{d} t\right] \\
& =\inf _{v_{2} \in \mathcal{U}_{2}} \sup _{v_{1} \in \mathcal{U}_{1}} \mathrm{E}_{x}\left[\int_{0}^{\tau_{R}} \mathrm{e}^{-\alpha t} h\left(X(t), v_{1}(t), v_{2}(t)\right) \mathrm{d} t\right] .
\end{aligned}
$$

It is evident that $\varphi_{\alpha}^{R}(x) \leq \tilde{\psi}_{\alpha}(x), x \in \mathbb{R}^{d}$, where

$$
\tilde{\psi}_{\alpha}(x):=\sup _{v_{1} \in \mathcal{U}_{1}} \inf _{v_{2} \in \mathcal{U}_{2}} \mathrm{E}_{x}\left[\int_{0}^{\infty} \mathrm{e}^{-\alpha t} h\left(X(t), v_{1}(t), v_{2}(t)\right) \mathrm{d} t\right], \quad x \in \mathbb{R}^{d} .
$$

Also $\varphi_{\alpha}^{R}$ is nondecreasing in $R$. By Assumption $\left(\mathrm{A} 3^{\prime}\right)$, it follows that

$$
\tilde{\psi}_{\alpha}(x) \leq k_{2} \mathrm{E}_{x}\left[\int_{0}^{\infty} \mathrm{e}^{-\alpha t} g(X(t)) \mathrm{d} t\right]
$$


where $X$ is a solution to (2.1) corresponding to some stationary Markov control pair. Since the function $x \mapsto \mathrm{E}_{x}\left[\int_{0}^{\infty} \mathrm{e}^{-\alpha t} g(X(t)) \mathrm{d} t\right]$ is continuous, it follows that $\tilde{\psi}_{\alpha} \in L_{l o c}^{p}\left(\mathbb{R}^{d}\right)$ for $1<p<\infty$.

Beneš' measurable selection theorem [4] asserts that there exist controls $\left(v_{1 \alpha}^{R}, v_{2 \alpha}^{R}\right) \in$ $\mathcal{M}_{1} \times \mathcal{M}_{2}$ which realize the minimax in (2.4)-(2.5), i.e., for all $x \in B_{R}$ the following holds: $\max _{v_{1} \in V_{1}} \min _{v_{2} \in V_{2}}\left[L \varphi_{\alpha}^{R}\left(x, v_{1}, v_{2}\right)+h\left(x, v_{1}, v_{2}\right)\right]=L \varphi_{\alpha}^{R}\left(x, v_{1 \alpha}^{R}(x), v_{2 \alpha}^{R}(x)\right)+h\left(x, v_{1 \alpha}^{R}(x), v_{2 \alpha}^{R}(x)\right)$. Hence $\varphi_{\alpha}^{R} \in C^{2}\left(B_{R}\right) \cap C\left(\overline{B_{R}}\right)$ is a solution to

$$
\alpha \varphi_{\alpha}^{R}(x)=L \varphi_{\alpha}^{R}\left(x, v_{1 \alpha}^{R}(x), v_{2 \alpha}^{R}(x)\right)+h\left(x, v_{1 \alpha}^{R}(x), v_{2 \alpha}^{R}(x)\right), \quad x \in B_{R} .
$$

Hence by [2, Lemma A.2.5, p. 305], for each $1<p<\infty$ and $R^{\prime}>2 R$, we have

$$
\begin{aligned}
\left\|\varphi_{\alpha}^{R^{\prime}}\right\|_{W^{2, p}\left(B_{R}\right)} & \leq K_{1}\left(\left\|\varphi_{\alpha}^{R^{\prime}}\right\|_{L^{p}\left(B_{2 R}\right)}+\left\|L \varphi_{\alpha}^{R^{\prime}}-\alpha \varphi_{\alpha}^{R^{\prime}}\right\|_{L^{p}\left(B_{2 R}\right)}\right) \\
& \leq K_{1}\left(\left\|\tilde{\psi}_{\alpha}\right\|_{L^{p}\left(B_{2 R}\right)}+\left\|h\left(\cdot, v_{1 \alpha}^{R^{\prime}}(\cdot), v_{2 \alpha}^{R^{\prime}}(\cdot)\right)\right\|_{L^{p}\left(B_{2 R}\right)}\right) \\
& \leq K_{1}\left(\left\|\tilde{\psi}_{\alpha}\right\|_{L^{p}\left(B_{2 R}\right)}+K_{2}(R)\left|B_{2 R}\right|^{1 / p}\right),
\end{aligned}
$$

where $K_{1}>0$ is a constant independent of $R^{\prime}$ and $K_{2}(R)$ is a constant depending only on the bound of $h$ on $B_{2 R}$. Using standard approximation arguments involving Sobolev imbedding theorems, see [2, p. 111], it follows that there exists $\psi_{\alpha} \in W_{l o c}^{2, p}\left(\mathbb{R}^{d}\right)$ such that $\varphi_{\alpha}^{R \uparrow} \psi_{\alpha}$ as $R \uparrow \infty$ and $\psi_{\alpha}$ is a solution to

$$
\alpha \psi_{\alpha}(x)=\max _{v_{1} \in V_{1}} \min _{v_{2} \in V_{2}}\left[L \psi_{\alpha}\left(x, v_{1}, v_{2}\right)+h\left(x, v_{1}, v_{2}\right)\right] .
$$

By standard regularity arguments, see [2, p. 109], one can show that $\psi_{\alpha} \in C^{2, r}\left(\mathbb{R}^{d}\right), 0<$ $r<1$. Also using the minimax condition, it follows that $\psi_{\alpha} \in C^{2, r}\left(\mathbb{R}^{d}\right), 0<r<1$, is a solution to

$$
\begin{aligned}
\alpha \psi_{\alpha}(x) & =\min _{v_{2} \in V_{2}} \max _{v_{1} \in V_{1}}\left[L \psi_{\alpha}\left(x, v_{1}, v_{2}\right)+h\left(x, v_{1}, v_{2}\right)\right] \\
& =\max _{v_{1} \in V_{1}} \min _{v_{2} \in V_{2}}\left[L \psi_{\alpha}\left(x, v_{1}, v_{2}\right)+h\left(x, v_{1}, v_{2}\right)\right] .
\end{aligned}
$$

Let $v_{1}^{\alpha} \in \mathcal{M}_{1}$ and $v_{2}^{\alpha} \in \mathcal{M}_{2}$ be an outer maximizing and an outer minimizing selector for (2.3) , respectively, corresponding to $\psi_{\alpha}$ given above. Then $\psi_{\alpha}$ satisfies the p.d.e.

$$
\alpha \psi_{\alpha}(x)=\max _{v_{1} \in V_{1}}\left[L \psi_{\alpha}\left(x, v_{1}, v_{2}^{\alpha}(x)\right)+h\left(x, v_{1}, v_{2}^{\alpha}(x)\right)\right] .
$$

For $v_{1} \in \mathcal{U}_{1}$, let $X$ be the solution to (2.1) corresponding to $\left(v_{1}, v_{2}^{\alpha}\right)$ and the initial condition $x \in \mathbb{R}^{d}$. Applying the Itô-Dynkin formula, we obtain

$$
\mathrm{E}_{x}\left[\mathrm{e}^{-\alpha \tau_{R}} \psi_{\alpha}\left(X\left(\tau_{R}\right)\right)\right]-\psi_{\alpha}(x) \leq-\mathrm{E}_{x}\left[\int_{0}^{\tau_{R}} \mathrm{e}^{-\alpha t} h\left(X(t), v_{1}(t), v_{2}^{\alpha}(X(t))\right) \mathrm{d} t\right] .
$$

Since $\psi_{\alpha} \geq 0$, we have

$$
\psi_{\alpha}(x) \geq \mathrm{E}_{x}\left[\int_{0}^{\tau_{R}} \mathrm{e}^{-\alpha t} h\left(X(t), v_{1}(t), v_{2}^{\alpha}(X(t))\right) \mathrm{d} t\right] .
$$


Using Fatou's lemma we obtain

$$
\psi_{\alpha}(x) \geq \mathrm{E}_{x}\left[\int_{0}^{\infty} \mathrm{e}^{-\alpha t} h\left(X(t), v_{1}(t), v_{2}^{\alpha}(X(t))\right) \mathrm{d} t\right] .
$$

Therefore

$$
\psi_{\alpha}(x) \geq \sup _{v_{1} \in \mathcal{U}_{1}} \mathrm{E}_{x}\left[\int_{0}^{\infty} \mathrm{e}^{-\alpha t} h\left(X(t), v_{1}(t), v_{2}^{\alpha}(X(t))\right) \mathrm{d} t\right] .
$$

Similarly, for $v_{2} \in \mathcal{U}_{2}$, let $X$ be the solution to (2.1) corresponding to $\left(v_{1}^{\alpha}, v_{2}\right)$ and the initial condition $x \in \mathbb{R}^{d}$. By applying the Itô-Dynkin formula, we obtain

$$
\mathrm{E}_{x}\left[\mathrm{e}^{-\alpha \tau_{R}} \psi_{\alpha}\left(X\left(\tau_{R}\right)\right)\right]-\psi_{\alpha}(x) \geq-\mathrm{E}_{x}\left[\int_{0}^{\tau_{R}} \mathrm{e}^{-\alpha t} h\left(X(t), v_{1}^{\alpha}(X(t)), v_{2}(t)\right) \mathrm{d} t\right] .
$$

Hence

$$
\psi_{\alpha}(x) \leq \mathrm{E}_{x}\left[\int_{0}^{\infty} \mathrm{e}^{-\alpha t} h\left(X(t), v_{1}^{\alpha}(X(t)), v_{2}(t)\right) \mathrm{d} t\right]+\mathrm{E}_{x}\left[\mathrm{e}^{-\alpha \tau_{R}} \psi_{\alpha}\left(X\left(\tau_{R}\right)\right)\right] .
$$

By [2, Remark A.3.8, p. 310], it follows that

$$
\lim _{R \uparrow \infty} \mathrm{E}_{x}\left[\mathrm{e}^{-\alpha \tau_{R}} \psi_{\alpha}\left(X\left(\tau_{R}\right)\right)\right]=0
$$

Hence, we have

$$
\psi_{\alpha}(x) \leq \mathrm{E}_{x}\left[\int_{0}^{\infty} \mathrm{e}^{-\alpha t} h\left(X(t), v_{1}^{\alpha}(X(t)), v_{2}(t)\right) \mathrm{d} t\right]
$$

Therefore

$$
\psi_{\alpha}(x) \leq \inf _{v_{2} \in \mathcal{U}_{2}} \mathrm{E}_{x}\left[\int_{0}^{\infty} \mathrm{e}^{-\alpha t} h\left(X(t), v_{1}^{\alpha}(X(t)), v_{2}(t)\right) \mathrm{d} t\right] .
$$

By (2.9) and (2.11), we obtain

$$
\psi_{\alpha}(x)=\mathrm{E}_{x}\left[\int_{0}^{\infty} \mathrm{e}^{-\alpha t} h\left(X(t), v_{1}^{\alpha}(X(t)), v_{2}^{\alpha}(X(t))\right) \mathrm{d} t\right] .
$$

Also by (2.8) and (2.10) we have

$$
\begin{aligned}
& \inf _{v_{2} \in \mathcal{U}_{2}} \sup _{v_{1} \in \mathcal{U}_{1}} \mathrm{E}_{x}\left[\int_{0}^{\infty} \mathrm{e}^{-\alpha t} h(X(t),\right.\left.\left.v_{1}(t), v_{2}(t)\right) \mathrm{d} t\right] \leq \psi_{\alpha}(x) \\
& \leq \sup _{v_{1} \in \mathcal{U}_{1}} \inf _{v_{2} \in \mathcal{U}_{2}} \mathrm{E}_{x}\left[\int_{0}^{\infty} \mathrm{e}^{-\alpha t} h\left(X(t), v_{1}(t), v_{2}(t)\right) \mathrm{d} t\right] .
\end{aligned}
$$

This implies the desired characterization.

Remark 2.1. Using Theorem 2.1, one can easily show that any pair of measurable outer maximizing and outer minimizing selectors of (2.3) is a saddle point equilibrium for the stochastic differential game with state dynamics given by (2.1) and with a discounted criterion under the running payoff function $h$. 
Theorem 2.2. Assume (A1), (A2) and $\left(\mathrm{A} 3^{\prime}\right)$. Then there exists a solution $\left(\beta, \varphi^{*}\right) \in$ $\mathbb{R} \times C_{\mathcal{V}}\left(\mathbb{R}^{d}\right) \cap C^{2}\left(\mathbb{R}^{d}\right)$ to the Isaac's equation

$$
\begin{aligned}
\beta & =\min _{v_{2} \in V_{2}} \max _{v_{1} \in V_{1}}\left[L \varphi^{*}\left(x, v_{1}, v_{2}\right)+h\left(x, v_{1}, v_{2}\right)\right] \\
& =\max _{v_{1} \in V_{1}} \min _{v_{2} \in V_{2}}\left[L \varphi^{*}\left(x, v_{1}, v_{2}\right)+h\left(x, v_{1}, v_{2}\right)\right], \\
\varphi^{*}(0) & =0
\end{aligned}
$$

such that $\beta$ is the value of the game.

Proof. For $\left(v_{1}, v_{2}\right) \in \mathcal{M}_{1} \times \mathcal{M}_{2}$, define

$$
J_{\alpha}\left(x, v_{1}, v_{2}\right):=\mathrm{E}_{x}\left[\int_{0}^{\infty} \mathrm{e}^{-\alpha t} h\left(X(t), v_{1}(X(t)), v_{2}(X(t))\right) \mathrm{d} t\right], \quad x \in \mathbb{R}^{d},
$$

where $X$ is a solution to (2.1) corresponding to $\left(v_{1}, v_{2}\right) \in \mathcal{M}_{1} \times \mathcal{M}_{2}$. Hence from (2.12), we have

$$
\psi_{\alpha}(x)=J_{\alpha}\left(x, v_{1}^{\alpha}, v_{2}^{\alpha}\right),
$$

where $\left(v_{1}^{\alpha}, v_{2}^{\alpha}\right) \in \mathcal{M}_{1} \times \mathcal{M}_{2}$ is a pair of measurable outer maximizing and outer minimizing selectors of (2.3). Using $\left(\mathrm{A} 3^{\prime}\right)$, it is easy to see that $\left(v_{1}^{\alpha}, v_{2}^{\alpha}\right)$ is a pair of stable stationary Markov controls. Hence by the arguments in the proof of [2, Theorem 3.7.4, pp. 128-131], we have the following estimates:

$$
\begin{gathered}
\left\|\psi_{\alpha}-\psi_{\alpha}(0)\right\|_{W^{2, p}\left(B_{R}\right)} \leq \frac{K_{3}}{\eta\left[v_{1}^{\alpha}, v_{2}^{\alpha}\right]\left(B_{R}\right)}\left(\frac{\beta\left[v_{1}^{\alpha}, v_{2}^{\alpha}\right]}{\eta\left[v_{1}^{\alpha}, v_{2}^{\alpha}\right]\left(B_{R}\right)}\right. \\
\left.+\max _{\left(x, v_{1}, v_{2}\right) \in B_{4 R} \times V_{1} \times V_{2}} h\left(x, v_{1}, v_{2}\right)\right), \\
\sup _{\left.x \in B_{R}\right)} \alpha \psi_{\alpha}(x) \leq K_{3}\left(\frac{\beta\left[v_{1}^{\alpha}, v_{2}^{\alpha}\right]}{\eta\left[v_{1}^{\alpha}, v_{2}^{\alpha}\right]\left(B_{R}\right)}+\max _{\left(x, v_{1}, v_{2}\right) \in B_{4 R} \times V_{1} \times V_{2}} h\left(x, v_{1}, v_{2}\right)\right)
\end{gathered}
$$

where $\eta\left[v_{1}^{\alpha}, v_{2}^{\alpha}\right]$ is the unique invariant probability measure of the process (2.1) corresponding to $\left(v_{1}^{\alpha}, v_{2}^{\alpha}\right)$ and

$$
\beta\left[v_{1}^{\alpha}, v_{2}^{\alpha}\right]:=\int_{\mathbb{R}^{d}} h\left(x, v_{1}^{\alpha}(x), v_{2}^{\alpha}(x)\right) \eta\left[v_{1}^{\alpha}, v_{2}^{\alpha}\right](\mathrm{d} x) .
$$

It follows from [2, Corollary 3.3.2, p. 97] that

$$
\sup _{\alpha>0} \beta\left[v_{1}^{\alpha}, v_{2}^{\alpha}\right]<\infty
$$

Also from [2, (2.6.9a); p. 69 and (3.3.9); p. 97] it follows that

$$
\inf _{\alpha>0} \eta\left[v_{1}^{\alpha}, v_{2}^{\alpha}\right]\left(B_{R}\right)>0 .
$$

Combining (2.14)-(2.17), we have

$$
\begin{aligned}
\left\|\psi_{\alpha}-\psi_{\alpha}(0)\right\|_{W^{2, p}\left(B_{R}\right)} & \leq K_{4}, \\
\sup _{x \in B_{R}} \alpha \psi_{\alpha}(x) & \leq K_{4},
\end{aligned}
$$

where $K_{4}>0$ is a constant independent of $\alpha>0$. 
Define

$$
\bar{\psi}_{\alpha}(x):=\psi_{\alpha}(x)-\psi_{\alpha}(0), \quad x \in \mathbb{R}^{d} .
$$

In view of (2.18), one can use the arguments in [2, Lemma 3.5.4, pp. 108-109] to show that along some sequence $\alpha_{n} \downarrow 0, \alpha_{n} \psi_{\alpha}(0)$ converges to a constant $\varrho$ and $\bar{\psi}_{\alpha_{n}}$ converges uniformly on compact sets to a function $\varphi^{*} \in C^{2}\left(\mathbb{R}^{d}\right)$, where the pair $\left(\varrho, \varphi^{*}\right)$ is a solution to the p.d.e.

$$
\begin{aligned}
\varrho & =\min _{v_{2} \in V_{2}} \max _{v_{1} \in V_{1}}\left[L \varphi^{*}\left(x, v_{1}, v_{2}\right)+h\left(x, v_{1}, v_{2}\right)\right], \\
\varphi^{*}(0) & =0 .
\end{aligned}
$$

Moreover, using the Isaac's condition, it follows that $\left(\varrho, \varphi^{*}\right) \in \mathbb{R} \times C^{2}\left(\mathbb{R}^{d}\right)$ satisfies (2.13) .

We claim that $\varphi^{*} \in o(\mathcal{V})$, i.e., $\frac{\varphi^{*}(x)}{\mathcal{V}(x)} \rightarrow 0$ as $\|x\| \rightarrow \infty$. To prove the claim let $\left(v_{1}^{*}, v_{2}^{*}\right) \in$ $\mathcal{M}_{1} \times \mathcal{M}_{2}$ be a pair of measurable outer maximizing and outer minimizing selectors of (2.13) corresponding to $\varphi^{*}$. Let $X$ be the solution to (2.1) under the control $\left(v_{1}^{*}, v_{2}^{*}\right)$. Then by an application of the Itô-Dynkin formula and the help of Fatou's lemma, we can show that for all $x \in \mathbb{R}^{d}$

$$
\varphi^{*}(x) \geq \mathrm{E}_{x}\left[\int_{0}^{\breve{\tau}_{r}}\left(h\left(X(t), v_{1}^{*}(X(t)), v_{2}^{*}(X(t))\right)-\varrho\right) \mathrm{d} t\right]+\min _{\|y\|=r} \varphi^{*}(y),
$$

where

$$
\breve{\tau}_{r}=\inf \{t \geq 0:\|X(t)\| \leq r\} .
$$

Let $v_{1}^{\alpha} \in \mathcal{M}_{1}$ be a measurable outer maximizing selector in (2.3). Then the function $\psi_{\alpha} \in C^{2, r}\left(\mathbb{R}^{d}\right)$ given in Theorem 2.1 satisfies the p.d.e.

$$
\alpha \psi_{\alpha}=\min _{v_{2} \in V_{2}}\left[L \psi_{\alpha}\left(x, v_{1}^{\alpha}(x), v_{2}\right)+h\left(x, v_{1}^{\alpha}(x), v_{2}\right)\right] .
$$

Let $X$ be the solution to (2.1) under the control $\left(v_{1}^{\alpha}, v_{2}\right)$, with $v_{2} \in \mathcal{U}_{2}$, and initial condition $x \in \mathbb{R}^{d}$. Then by applying the Itô-Dynkin formula to $\mathrm{e}^{-\alpha t} \psi_{\alpha}(X(t))$ and using (2.20), we obtain

$$
\mathrm{E}_{x}\left[\mathrm{e}^{-\alpha\left(\breve{\tau}_{r} \wedge \tau_{R}\right)} \psi_{\alpha}\left(X\left(\breve{\tau}_{r} \wedge \tau_{R}\right)\right)\right]-\psi_{\alpha}(x) \geq-\mathrm{E}_{x}\left[\int_{0}^{\breve{\tau}_{r} \wedge \tau_{R}} h\left(X(t), v_{1}^{\alpha}(X(t)), v_{2}(t)\right) \mathrm{d} t\right],
$$

which we write as

$$
\psi_{\alpha}(x) \leq \mathrm{E}_{x}\left[\int_{0}^{\breve{\tau}_{r}} h\left(X(t), v_{1}^{\alpha}(X(t)), v_{2}(t)\right) \mathrm{d} t\right]+\mathrm{E}_{x}\left[\mathrm{e}^{-\alpha\left(\breve{\tau}_{r} \wedge \tau_{R}\right)} \psi_{\alpha}\left(X\left(\breve{\tau}_{r} \wedge \tau_{R}\right)\right)\right] .
$$

Using [2, Remark A.3.8, p. 310], it follows that

$$
\mathrm{E}_{x}\left[\mathrm{e}^{-\alpha \tau_{R}} \psi_{\alpha}\left(X\left(\tau_{R}\right)\right) I\left\{\breve{\tau}_{r} \geq \tau_{R}\right\}\right] \leq \mathrm{E}_{x}\left[\mathrm{e}^{-\alpha \tau_{R}} \psi_{\alpha}\left(X\left(\tau_{R}\right)\right)\right] \underset{R \rightarrow \infty}{\longrightarrow} 0 .
$$

Hence from (2.21) and (2.22), we obtain

$$
\psi_{\alpha}(x) \leq \mathrm{E}_{x}\left[\int_{0}^{\breve{\tau}_{r}} h\left(X(t), v_{1}^{\alpha}(X(t)), v_{2}(t)\right) \mathrm{d} t\right]+\mathrm{E}_{x}\left[\mathrm{e}^{-\alpha \breve{\tau}_{r}} \psi_{\alpha}\left(X\left(\breve{\tau}_{r}\right)\right)\right] .
$$


Therefore,

$$
\begin{aligned}
\bar{\psi}_{\alpha}(x) \leq \mathrm{E}_{x}\left[\int_{0}^{\breve{\tau_{r}}} h\left(X(t), v_{1}^{\alpha}(X(t)), v_{2}(t)\right) \mathrm{d} t\right]+\mathrm{E}_{x}\left[\mathrm{e}^{-\alpha \breve{\tau}_{r}} \psi_{\alpha}\left(X\left(\breve{\tau}_{r}\right)\right)-\psi_{\alpha}(0)\right] \\
=\mathrm{E}_{x}\left[\int_{0}^{\breve{\tau}_{r}}\left(h\left(X(t), v_{1}^{\alpha}(X(t)), v_{2}(t)\right)-\varrho\right) \mathrm{d} t\right]+\mathrm{E}_{x}\left[\psi_{\alpha}\left(X\left(\breve{\tau}_{r}\right)\right)-\psi_{\alpha}(0)\right] \\
\quad+\mathrm{E}_{x}\left[\alpha^{-1}\left(1-\mathrm{e}^{-\alpha \breve{\tau}_{r}}\right)\left(\varrho-\alpha \psi_{\alpha}\left(X\left(\breve{\tau}_{r}\right)\right)\right]\right. \\
\leq \mathrm{E}_{x}\left[\int_{0}^{\breve{\tau}_{r}}\left(h\left(X(t), v_{1}^{\alpha}(X(t)), v_{2}(t)\right)-\varrho\right) \mathrm{d} t\right]+M(r) \\
\quad+\mathrm{E}_{x}\left[\breve{\tau}_{r}\right] \sup _{\|y\|=r}\left|\varrho-\alpha \psi_{\alpha}(y)\right| \\
\leq \sup _{v_{1} \in \mathcal{M}_{1}} \mathrm{E}_{x}\left[\int_{0}^{\breve{\tau}_{r}}\left(h\left(X(t), v_{1}(X(t)), v_{2}(t)\right)-\varrho\right) \mathrm{d} t\right] \\
+M(r)+\sup _{\|y\|=r}\left|\varrho-\alpha \psi_{\alpha}(y)\right| \sup _{v_{1} \in \mathcal{M}_{1}} \mathrm{E}_{x}\left[\breve{\tau}_{r}\right]
\end{aligned}
$$

for some nonnegative constant $M(r)$ such that $M(r) \rightarrow 0$ as $r \downarrow 0$. Next from the definition of $\varphi^{*}$, by letting $\alpha \downarrow 0$ along the sequence given in the proof of Theorem 2.2, we obtain

$$
\varphi^{*}(x) \leq \sup _{v_{1} \in \mathcal{M}_{1}} \mathrm{E}_{x}\left[\int_{0}^{\breve{\tau}_{r}}\left(h\left(X(t), v_{1}(X(t)), v_{2}(t)\right)-\varrho\right) \mathrm{d} t\right]+M(r) .
$$

By combining (2.19) and (2.23), the result follows by [2, Lemma 3.7.2, p. 125]. This completes the proof of the claim.

Let $\left(v_{1}^{*}, v_{2}^{*}\right) \in \mathcal{M}_{1} \times \mathcal{M}_{2}$ be a pair of measurable outer maximizing and minimizing selectors in (2.13) corresponding to $\varphi^{*}$. Then $\left(\varrho, \varphi^{*}\right)$ satisfies the p.d.e.

$$
\varrho=\max _{v_{1} \in V_{1}}\left[L \varphi^{*}\left(x, v_{1}, v_{2}^{*}(x)\right)+h\left(x, v_{1}, v_{2}^{*}(x)\right)\right] .
$$

Let $v_{1} \in \mathcal{U}_{1}$ and $X$ be the process in (2.1) under the control $\left(v_{1}, v_{2}^{*}\right)$ and initial condition $x \in \mathbb{R}^{d}$. By applying the Itô-Dynkin formula, we obtain

$$
\mathrm{E}_{x}\left[\varphi^{*}\left(X\left(t \wedge \tau_{R}\right)\right)\right]-\varphi^{*}(x) \leq-\mathrm{E}_{x}\left[\int_{0}^{t \wedge \tau_{R}}\left(h\left(X(t), v_{1}(t), v_{2}^{*}(X(t))\right)-\varrho\right) \mathrm{d} t\right] .
$$

Hence

$$
\varrho t \geq \mathrm{E}_{x}\left[\int_{0}^{t \wedge \tau_{R}} h\left(X(t), v_{1}(t), v_{2}^{*}(X(t))\right) \mathrm{d} t\right]+\mathrm{E}_{x}\left[\varphi^{*}\left(X\left(t \wedge \tau_{R}\right)\right)\right]-\varphi^{*}(x)
$$

for all $t \geq 0$. Using Fatou's lemma and [2, Lemma 3.7.2, p. 125], we obtain

$$
\varrho t \geq \mathrm{E}_{x}\left[\int_{0}^{t} h\left(X(t), v_{1}(t), v_{2}^{*}(X(t))\right) \mathrm{d} t\right]+\mathrm{E}_{x}\left[\varphi^{*}(X(t))\right]-\varphi^{*}(x), \quad t \geq 0 .
$$


Dividing by $t$ and taking limits again using [2, Lemma 3.7.2, p. 125], we obtain

$$
\varrho \geq \liminf _{t \rightarrow \infty} \frac{1}{t} \mathrm{E}_{x}\left[\int_{0}^{t} h\left(X(t), v_{1}(t), v_{2}^{*}(X(t))\right) \mathrm{d} t\right] .
$$

Since $v_{1} \in \mathcal{U}_{1}$ was arbitrary, we have

$$
\begin{aligned}
\varrho & \geq \sup _{v_{1} \in \mathcal{U}_{1}} \liminf _{t \rightarrow \infty} \frac{1}{t} \mathrm{E}_{x}\left[\int_{0}^{t} h\left(X(t), v_{1}(t), v_{2}^{*}(X(t))\right) \mathrm{d} t\right] \\
& \geq \inf _{v_{2} \in \mathcal{U}_{2}} \sup _{v_{1} \in \mathcal{U}_{1}} \liminf _{t \rightarrow \infty} \frac{1}{t} \mathrm{E}_{x}\left[\int_{0}^{t} h\left(X(t), v_{1}(t), v_{2}(t)\right) \mathrm{d} t\right] .
\end{aligned}
$$

The pair $\left(\varrho, \varphi^{*}\right)$ also satisfies the p.d.e.

$$
\varrho=\min _{v_{2} \in V_{2}}\left[L \varphi^{*}\left(x, v_{1}^{*}(x), v_{2}\right)+h\left(x, v_{1}^{*}(x), v_{2}\right)\right] .
$$

Let $v_{2} \in \mathcal{U}_{2}$ and $X$ be the process in (2.1) corresponding to $\left(v_{1}^{*}, v_{2}\right)$ and initial condition $x \in \mathbb{R}^{d}$. By applying the Itô-Dynkin formula, we obtain

Hence

$$
\mathrm{E}_{x}\left[\varphi^{*}\left(X\left(t \wedge \tau_{R}\right)\right)\right]-\varphi^{*}(x) \geq-\mathrm{E}_{x}\left[\int_{0}^{t \wedge \tau_{R}}\left(h\left(X(t), v_{1}^{*}(X(t)), v_{2}(t)\right)-\varrho\right) \mathrm{d} t\right] .
$$

$$
\varrho \mathrm{E}_{x}\left[t \wedge \tau_{R}\right] \leq \mathrm{E}_{x}\left[\int_{0}^{t} h\left(X(t), v_{1}^{*}(X(t)), v_{2}(t)\right) \mathrm{d} t+\varphi^{*}\left(X\left(t \wedge \tau_{R}\right)\right)\right]-\varphi^{*}(x) .
$$

Next, by letting $R \rightarrow \infty$ and using the dominated convergence theorem for the l.h.s. and [2, Lemma 3.7.2, p. 125] for the r.h.s., we obtain

$$
\varrho t \leq \mathrm{E}_{x}\left[\int_{0}^{t} h\left(X(t), v_{1}^{*}(X(t)), v_{2}(t)\right) \mathrm{d} t\right]+\mathrm{E}_{x}\left[\varphi^{*}(X(t))\right]-\varphi^{*}(x) .
$$

Also by [2, Lemma 3.7.2, p. 125], we obtain

$$
\varrho \leq \liminf _{t \rightarrow \infty} \frac{1}{t} \mathrm{E}_{x}\left[\int_{0}^{t} h\left(X(t), v_{1}^{*}(X(t)), v_{2}(t)\right) \mathrm{d} t\right] .
$$

Since $v_{2} \in \mathcal{U}_{2}$ was arbitrary, we have

$$
\begin{aligned}
\varrho & \leq \inf _{v_{2} \in \mathcal{U}_{2}} \liminf _{t \rightarrow \infty} \frac{1}{t} \mathrm{E}_{x}\left[\int_{0}^{t} h\left(X(t), v_{1}^{*}(X(t)), v_{2}(t)\right) \mathrm{d} t\right] \\
& \leq \sup _{v_{1} \in \mathcal{U}_{1}} \inf _{v_{2} \in \mathcal{U}_{2}} \liminf _{t \rightarrow \infty} \frac{1}{t} \mathrm{E}_{x}\left[\int_{0}^{t} h\left(X(t), v_{1}(t), v_{2}(t)\right) \mathrm{d} t\right] .
\end{aligned}
$$

Combining (2.24) and (2.25), we obtain

$$
\begin{aligned}
\varrho & =\inf _{v_{2} \in \mathcal{U}_{2}} \sup _{v_{1} \in \mathcal{U}_{1}} \liminf _{t \rightarrow \infty} \frac{1}{t} \mathrm{E}_{x}\left[\int_{0}^{t} h\left(X(t), v_{1}(t), v_{2}(t)\right) \mathrm{d} t\right] \\
& =\sup _{v_{1} \in \mathcal{U}_{1}} \inf _{v_{2} \in \mathcal{U}_{2}} \liminf _{t \rightarrow \infty} \frac{1}{t} \mathrm{E}_{x}\left[\int_{0}^{t} h\left(X(t), v_{1}(t), v_{2}(t)\right) \mathrm{d} t\right],
\end{aligned}
$$

i.e. $\varrho=\beta$, the value of the game. This completes the proof. 
Remark 2.2. Using Theorem 2.2, one can easily prove that any pair of measurable outer maximizing and outer minimizing selectors of (2.3) is a saddle point equilibrium for the stochastic differential game with state dynamics given by (2.1) and with the ergodic criterion under the running payoff function $h$.

The following corollary, stated here without proof, follows along the lines of the proof of [2, Theorem 3.7.12].

Corollary 2.1. The solution $\varphi^{*}$ has the stochastic representation

$$
\begin{aligned}
\varphi^{*}(x) & =\lim _{r \downarrow 0} \sup _{v_{1} \in \mathcal{M}_{1}} \inf _{v_{2} \in \mathcal{M}_{2}} \mathrm{E}_{x}\left[\int_{0}^{\breve{\tau}_{r}}\left(h\left(X(t), v_{1}(X(t)), v_{2}(X(t))\right)-\beta\right) \mathrm{d} t\right] \\
& =\lim _{r \downarrow 0} \inf _{v_{2} \in \mathcal{M}_{2}} \sup _{v_{1} \in \mathcal{M}_{1}} \mathrm{E}_{x}\left[\int_{0}^{\breve{\tau_{r}}}\left(h\left(X(t), v_{1}(X(t)), v_{2}(X(t))\right)-\beta\right) \mathrm{d} t\right] \\
& =\lim _{r \downarrow 0} \mathrm{E}_{x}\left[\int_{0}^{\breve{\tau}_{r}}\left(h\left(X(t), v_{1}^{*}(X(t)), v_{2}^{*}(X(t))\right)-\beta\right) \mathrm{d} t\right] .
\end{aligned}
$$

and is unique in the class of functions that do not grow faster than $\mathcal{V}$ and vanish at $x=0$.

\section{Relative Value Iteration}

We consider the following relative value iteration equation.

$$
\begin{aligned}
\frac{\partial \varphi}{\partial t}(t, x) & =\min _{v_{2} \in V_{2}} \max _{v_{1} \in V_{1}}\left[L \varphi\left(t, x, v_{1}, v_{2}\right)+h\left(x, v_{1}, v_{2}\right)\right]-\varphi(t, 0), \\
\varphi(0, x) & =\varphi_{0}(x),
\end{aligned}
$$

where $\varphi_{0} \in C_{\mathcal{V}}\left(\mathbb{R}^{d}\right) \cap C^{2}\left(\mathbb{R}^{d}\right)$. This can be viewed as a continuous time continuous state space variant of the relative value iteration algorithm for Markov decision processes [12].

Convergence of this relative value iteration scheme is obtained through the study of the value iteration equation which takes the form

$$
\begin{aligned}
\frac{\partial \bar{\varphi}}{\partial t}(t, x) & =\min _{v_{2} \in V_{2}} \max _{v_{1} \in V_{1}}\left[L \bar{\varphi}\left(t, x, v_{1}, v_{2}\right)+h\left(x, v_{1}, v_{2}\right)\right]-\beta, \\
\bar{\varphi}(0, x) & =\varphi_{0}(x),
\end{aligned}
$$

where $\beta$ is the value of the average payoff game in Theorem 2.2 ,

Under Assumption (A3), it is straightforward to show that for each $T>0$ there exists a unique solution $\bar{\varphi}$ in $C_{\mathcal{V}}\left([0, T] \times \mathbb{R}^{d}\right) \cap C^{1,2}\left([0, T] \times \mathbb{R}^{d}\right)$ to the p.d.e. (3.2).

First, we prove the following important estimate which is crucial for the proof of convergence.

Lemma 3.1. Assume (A1)-(A3). Then for each $T>0$, the p.d.e. in (3.1) has a unique solution $\varphi \in C_{\mathcal{V}}\left([0, T] \times \mathbb{R}^{d}\right) \cap C^{1,2}\left([0, T] \times \mathbb{R}^{d}\right)$.

Proof. The proof follows by mimicking the arguments in [1, Lemma 4.1], using the following estimate

$$
\mathrm{E}_{x}[\mathcal{V}(X(t))] \leq \frac{k_{0}}{2 k_{1}}+\mathcal{V}(x) \mathrm{e}^{-2 k_{1} t}
$$


where $X$ is the solution to (2.1) corresponding to any admissible controls $v_{1}$ and $v_{2}$ and initial condition $x \in \mathbb{R}^{d}$. The estimate for $\varphi$ follows from the arguments in [2, Lemma 2.5.5, pp. 63-64], noting that for all $v_{i} \in \mathcal{U}_{i}, i=1,2$, we have

$$
\begin{aligned}
\int_{0}^{t} \mathrm{E}_{x}\left[h^{n}\left(X(s), v_{1}(s), v_{2}(s)\right)\right] \mathrm{d} s & \leq k_{2} \int_{0}^{t} \mathrm{E}_{x}[\mathcal{V}(X(s))] \mathrm{d} s \\
& \leq \frac{k_{2}}{2 k_{1}}\left(k_{0} t+\mathcal{V}(x)\right),
\end{aligned}
$$

where $h^{n}\left(x, v_{1}, v_{2}\right):=n \wedge h\left(x, v_{1}, v_{2}\right)$ is the truncation of $h$ at $n \geq 0$.

Next, we turn our attention to the p.d.e. in (3.2). It is straightforward to show that the solution $\bar{\varphi}$ to (3.2) also satisfies

$$
\begin{aligned}
\frac{\partial \bar{\varphi}}{\partial t}(t, x) & =\max _{v_{1} \in V_{1}} \min _{v_{2} \in V_{2}}\left[L \bar{\varphi}\left(t, x, v_{1}, v_{2}\right)+h\left(x, v_{1}, v_{2}\right)\right]-\beta, \\
\bar{\varphi}(0, x) & =\varphi_{0}(x),
\end{aligned}
$$

Definition 3.1. We let $\bar{v}_{i}: \mathbb{R}_{+} \times \mathbb{R}^{d} \rightarrow V_{i}$ for $i=1,2$ be an outer maximizing and an outer minimizing selector of (3.4) and (3.2), respectively. For each $t \geq 0$ we define the (nonstationary) Markov control

$$
\bar{v}_{i}^{t}:=\left\{\bar{v}_{i}^{t}(s, \cdot)=\bar{v}_{i}(t-s, \cdot), s \in[0, t]\right\} .
$$

We also let $\mathrm{P}_{x}^{v_{1}, v_{2}}$ denote the probability measure and $\mathrm{E}_{x}^{v_{1}, v_{2}}$ the expectation operator on the canonical space of the process under the control $v_{i} \in \mathcal{U}_{i}, i=1,2$, conditioned on the process $X$ starting from $x \in \mathbb{R}^{d}$ at $t=0$.

It is straightforward to show that the solution $\bar{\varphi}$ of (3.2) satisfies,

$$
\begin{aligned}
& \bar{\varphi}(t, x)=\mathrm{E}_{x}^{\bar{v}_{1}^{t}, \bar{v}_{2}^{t}}\left[\int_{0}^{t-s}\left(h\left(X(\tau), \bar{v}_{1}(t-\tau, X(\tau)), \bar{v}_{2}(t-\tau, X(\tau))\right)-\beta\right) \mathrm{d} \tau\right. \\
& +\bar{\varphi}(s, X(t-s))] \\
& =\inf _{v_{2} \in \mathcal{U}_{2}} \sup _{v_{1} \in \mathcal{U}_{1}} \mathrm{E}_{x}^{v_{1}, v_{2}}\left[\int_{0}^{t-s}\left(h\left(X(\tau), v_{1}(\tau), v_{2}(\tau)\right)-\beta\right) \mathrm{d} \tau+\bar{\varphi}(s, X(t-s))\right] \\
& =\sup _{v_{1} \in \mathcal{U}_{1}} \inf _{v_{2} \in \mathcal{U}_{2}} \mathrm{E}_{x}^{v_{1}, v_{2}}\left[\int_{0}^{t-s}\left(h\left(X(\tau), v_{1}(\tau), v_{2}(\tau)\right)-\beta\right) \mathrm{d} \tau\right. \\
& +\bar{\varphi}(s, X(t-s))]
\end{aligned}
$$

for all $t \geq s \geq 0$.

Lemma 3.2. Assume (A1)-(A3). For each $\varphi_{0} \in C_{\mathcal{V}}\left(\mathbb{R}^{d}\right) \cap C^{2}\left(\mathbb{R}^{d}\right)$, the solution $\bar{\varphi}$ of the p.d.e. (3.2) satisfies the following estimate

$$
\left|\bar{\varphi}(t, x)-\varphi^{*}(x)\right| \leq\left\|\bar{\varphi}(s, \cdot)-\varphi^{*}\right\|_{\mathcal{V}}\left(\frac{k_{0}}{2 k_{1}}+\mathcal{V}(x) \mathrm{e}^{-2 k_{1}(t-s)}\right) \quad \forall x \in \mathbb{R}^{d},
$$


and for all $t \geq s \geq 0$, where $\varphi^{*}$ is as in Theorem 2.2.

Proof. Let $v_{1}^{*} \in \mathcal{M}_{1}$ and $v_{2}^{*} \in \mathcal{M}_{2}$ be an outer maximizing and outer minimizing selector of (2.13), respectively. By (3.5) we obtain

$$
\bar{\varphi}(t, x)-\varphi^{*}(x) \leq \mathrm{E}_{x}^{\bar{v}_{1}^{t}, v_{2}^{*}}\left[\bar{\varphi}(s, X(t-s))-\varphi^{*}(X(t-s))\right]
$$

and

$$
\varphi^{*}(x)-\bar{\varphi}(t, x) \leq \mathrm{E}_{x}^{v_{1}^{*}, \bar{v}_{2}^{t}}\left[\varphi^{*}(X(t-s))-\bar{\varphi}(s, X(t-s))\right]
$$

for all $t \geq s \geq 0$. By (3.6)-(3.7) we obtain

$$
\left|\bar{\varphi}(t, x)-\varphi^{*}(x)\right| \leq \sup _{\left(v_{1}, v_{2}\right) \in \mathcal{U}_{1} \times \mathcal{U}_{2}} \mathrm{E}_{x}^{v_{1}, v_{2}}\left[\left|\bar{\varphi}(s, X(t-s))-\varphi^{*}(X(t-s))\right|\right],
$$

and an application of (3.3) completes the proof.

Arguing as in the proof of [1, Lemma 4.4], we can show the following:

Lemma 3.3. Assume (A1)-(A3). If $\bar{\varphi}(0, x)=\varphi(0, x)=\varphi_{0}(x)$ for some $\varphi_{0} \in C_{\mathcal{V}}\left(\mathbb{R}^{d}\right) \cap$ $C^{2}\left(\mathbb{R}^{d}\right)$, then

$$
\varphi(t, x)-\varphi(t, 0)=\bar{\varphi}(t, x)-\bar{\varphi}(t, 0)
$$

and

$$
\varphi(t, x)=\bar{\varphi}(t, x)-\mathrm{e}^{-t} \int_{0}^{t} \mathrm{e}^{s} \bar{\varphi}(s, 0) \mathrm{d} s+\beta\left(1-\mathrm{e}^{-t}\right)
$$

for all $x \in \mathbb{R}^{d}$ and $t \geq 0$.

Convergence of the relative value iteration is asserted in the following theorem.

Theorem 3.1. Assume (A1)-(A3). For each $\varphi_{0} \in C_{\mathcal{V}}\left(\mathbb{R}^{d}\right) \cap C^{2}\left(\mathbb{R}^{d}\right), \bar{\varphi}(t, x)$ converges to $\varphi^{*}(x)+$ constant and $\varphi(t, x)$ converges to $\varphi^{*}(x)+\beta$ as $t \rightarrow \infty$.

Proof. By Lemma 3.2 the map $x \mapsto \bar{\varphi}(t, x)$ is locally bounded, uniformly in $t \geq 0$. It then follows that $\left\{\frac{\partial^{2} \bar{\varphi}(t, x)}{\partial x_{i} \partial x_{j}}, t \geq 1\right\}$ are locally Hölder equicontinuous (see [10, Theorem 5.1]). Therefore the $\omega$-limit set $\omega\left(\varphi_{0}\right)$ of any initial condition $\varphi_{0} \in C_{\mathcal{V}}\left(\mathbb{R}^{d}\right) \cap C^{2}\left(\mathbb{R}^{d}\right)$ is a nonempty compact subset of $C_{\mathcal{V}}\left(\mathbb{R}^{d}\right) \cap C_{l o c}^{2}\left(\mathbb{R}^{d}\right)$.

To simplify the notation we define

$$
\Phi_{t}(x):=\bar{\varphi}(t, x)-\varphi^{*}(x), \quad(t, x) \in \mathbb{R}_{+} \times \mathbb{R}^{d} .
$$

By Lemma 3.2, if $f \in \omega\left(\varphi_{0}\right)$ then

$$
\limsup _{t \rightarrow \infty}\left|\Phi_{t}(x)\right| \leq \frac{k_{0}}{2 k_{1}}\left\|\varphi_{0}-\varphi^{*}\right\|_{\mathcal{V}} .
$$

Let $\left\{t_{n} \quad n \in \mathbb{N}\right\} \subset \mathbb{R}_{+}$be any increasing sequence such that $t_{n} \uparrow \infty$ and

$$
\Phi_{t_{n}} \rightarrow f \in C_{\mathcal{V}}\left(\mathbb{R}^{d}\right) \cap C^{2}\left(\mathbb{R}^{d}\right) \quad \text { as } n \rightarrow \infty .
$$

Dropping to a subsequence we assume that $t_{n+1}-t_{n} \uparrow \infty$ as $n \rightarrow \infty$. By construction $f+\varphi^{*} \in \omega\left(\varphi_{0}\right)$. 
We first show that $f$ is a constant. We define

$$
\bar{f}:=\sup _{x \in \mathbb{R}^{d}} f(x)
$$

and a subsequence $\left\{k_{n}\right\} \subset \mathbb{N}$ by

$$
k_{n}:=\sup \left\{k \in \mathbb{N}: \sup _{x \in B_{k}} \Phi_{t_{n}}(x) \leq \bar{f}+\frac{1}{k}\right\} .
$$

Since $\Phi_{t_{n}}$ converges to $f$ uniformly on compact sets as $n \rightarrow \infty$, it follows that $k_{n} \uparrow \infty$ as $n \rightarrow \infty$. Let $D$ be any fixed closed ball centered at the origin such that

$$
\inf _{x \in D^{c}} \mathcal{V}(x) \geq \frac{2 k_{0}\left\|\varphi_{0}-\varphi^{*}\right\|_{\mathcal{V}}}{k_{1}}
$$

It is straightforward to verify using (3.3) that if $X$ is the solution to (2.1) corresponding to any admissible controls $v_{1}$ and $v_{2}$ and initial condition $x \in \mathbb{R}^{d}$ then there exists $T_{0}<\infty$ depending only on $x$, such that

$$
\mathrm{P}_{x}\left(X_{t} \in D\right) \geq \frac{1}{2} \quad \forall x \in \mathbb{R}^{d}, \quad \forall t \geq T_{0}(x) .
$$

By the standard estimates of hitting probabilities for diffusions (see [9, Lemma 1.1]) for any $r>0$ there exists a constant $\gamma>0$ depending only on $r$ and $D$, such that with $B_{r}(y)$ denoting the open ball of radius $r$ centered at $y \in \mathbb{R}^{d}$ we have

$$
\mathrm{P}_{x}\left(X_{t} \in B_{r}(y)\right) \geq \gamma \quad \forall t \in[0,1], \quad \forall x, y \in D .
$$

Let $\mathrm{I}_{A}(\cdot)$ denote the indicator function of a set $A \subset \mathbb{R}^{d}$. An equivalent statement to (3.10) is that if $g: D \rightarrow \mathbb{R}_{+}$is a Hölder continuous function then there exists a continuous function $\Gamma: \mathbb{R}_{+} \rightarrow \mathbb{R}_{+}$, satisfying $\Gamma(z)>0$ for $z>0$ and depending only on $D$ and the Hölder constant of $g$, such that

$$
\mathrm{E}_{x}\left(g\left(X_{t}\right) \mathrm{I}_{D}\left(X_{t}\right)\right) \geq \Gamma\left(\max _{y \in D} g(y)\right) \quad \forall t \in[0,1], \quad \forall x \in D .
$$

Combining (3.9) and (3.11) and using the Markov property, we obtain

$$
\begin{aligned}
\mathrm{E}_{x}\left[g\left(X_{t}\right) \mathrm{I}_{D}\left(X_{t}\right)\right] & \geq \mathrm{E}_{x}\left[\mathrm{E}_{X_{t-1}}\left[g\left(X_{1}\right) \mathrm{I}_{D}\left(X_{1}\right)\right] \mathrm{I}_{D}\left(X_{t-1}\right)\right] \\
& \geq \Gamma\left(\max _{y \in D} g(y)\right) \mathrm{P}_{x}\left(X_{t-1} \in D\right) \\
& \geq \frac{1}{2} \Gamma\left(\max _{y \in D} g(y)\right) \quad \forall t \geq T_{0}(x)+1 .
\end{aligned}
$$

and for all $x \in \mathbb{R}^{d}$. Note that if $n$ is sufficiently large, then $D \subset B_{k_{n}}$ and therefore the function $x \mapsto \bar{f}+\frac{1}{k_{n}}-\Phi_{t_{n}}(x)$ is nonnegative on $D$. Thus the local Hölder equicontinuity of $\left\{\Phi_{t}, t>0\right\}$ (this collection of functions locally share a common Hölder exponent) allows 
us to apply (3.12) for any fixed $x \in \mathbb{R}^{d}$ to obtain

$$
\begin{aligned}
\mathrm{E}_{x}^{\bar{v}_{1}^{t_{n+1}}, v_{2}^{*}}\left[\left(\bar{f}+\frac{1}{k_{n}}-\Phi_{t_{n}}\left(X\left(t_{n+1}-t_{n}\right)\right)\right) \mathrm{I}_{D}\left(X\left(t_{n+1}-t_{n}\right)\right)\right] & \\
& \geq \frac{1}{2} \Gamma\left(\bar{f}+\frac{1}{k_{n}}-\min _{y \in D} \Phi_{t_{n}}(y)\right),
\end{aligned}
$$

for all $n$ large enough. For $A \subset \mathbb{R}^{d}$ and $x \in \mathbb{R}^{d}$ we define

$$
\Psi_{n}(x ; A):=\mathrm{E}_{x}^{\bar{v}_{1}^{t_{n+1}}, v_{2}^{*}}\left[\Phi_{t_{n}}\left(X\left(t_{n+1}-t_{n}\right)\right) \mathrm{I}_{A}\left(X\left(t_{n+1}-t_{n}\right)\right)\right]
$$

By (3.6), (3.8) and (3.13) we have

$$
\begin{aligned}
\Phi_{t_{n+1}}(x) \leq & \mathrm{E}_{x}^{\bar{v}_{n+1}^{t_{n+1}}, v_{2}^{*}}\left[\Phi_{t_{n}}\left(X\left(t_{n+1}-t_{n}\right)\right)\right] \\
= & \Psi_{n}(x ; D)+\Psi_{n}\left(x ; B_{k_{n}} \backslash D\right)+\Psi_{n}\left(x ; B_{k_{n}}^{c}\right) \\
\leq & \left(\bar{f}+\frac{1}{k_{n}}\right) \mathrm{E}_{x}^{\bar{v}_{1}^{t_{n+1}}, v_{2}^{*}}\left[\mathrm{I}_{B_{k_{n}}}\left(X\left(t_{n+1}-t_{n}\right)\right)\right] \\
& \quad-\frac{1}{2} \Gamma\left(\bar{f}+\frac{1}{k_{n}}-\min _{y \in D} \Phi_{t_{n}}(y)\right)+\Psi_{n}\left(x ; B_{k_{n}}^{c}\right) \\
\leq & \bar{f}+\frac{1}{k_{n}}-\frac{1}{2} \Gamma\left(\bar{f}+\frac{1}{k_{n}}-\min _{y \in D} \Phi_{t_{n}}(y)\right)+\Psi_{n}\left(x ; B_{k_{n}}^{c}\right) .
\end{aligned}
$$

We claim that $\Psi_{n}\left(x ; B_{k_{n}}^{c}\right) \rightarrow 0$ as $n \rightarrow \infty$. Indeed if $X$ is the solution to (2.1) corresponding to any admissible controls $v_{1}$ and $v_{2}$ and initial condition $x \in \mathbb{R}^{d}$ then by (3.3) we have

$$
\mathrm{E}_{x}\left[\Phi_{t}(X(s)) \mathrm{I}_{B_{R}^{c}}(X(s))\right] \leq\left\|\Phi_{t} \mathrm{I}_{B_{R}^{c}}\right\|_{\mathcal{V}}\left(\frac{k_{0}}{2 k_{1}}+\mathcal{V}(x) \mathrm{e}^{-2 k_{1} s}\right)
$$

By Lemma 3.2 we have

$$
\left\|\Phi_{t} \mathrm{I}_{B_{R}^{c}}\right\|_{\mathcal{V}} \leq\left\|\Phi_{0}\right\|_{\mathcal{V}}\left(\frac{k_{0}}{2 k_{1} \inf _{x \in B_{R}^{c}} \mathcal{V}(x)}+\mathrm{e}^{-2 k_{1} t}\right) .
$$

It follows by (3.15) $-(3.16)$ that

$$
\mathrm{E}_{x}\left[\Phi_{t}(X(s)) \mathrm{I}_{B_{R}^{c}}(X(s))\right] \underset{\min \{t, R\} \rightarrow \infty}{\longrightarrow} 0
$$

uniformly in $s \geq 0$, which proves that $\Psi_{n}\left(x ; B_{k_{n}}^{c}\right) \rightarrow 0$ as $n \rightarrow \infty$. Thus, taking limits as $n \rightarrow \infty$ in (3.14), we obtain

$$
f(x) \leq \bar{f}-\frac{1}{2} \Gamma\left(\bar{f}-\min _{y \in D} f(y)\right) \quad \forall x \in \mathbb{R}^{d} .
$$

Taking the supremum over $x \in \mathbb{R}^{d}$ of the left hand side of (3.17) it follows that

$$
\Gamma\left(\bar{f}-\min _{y \in D} f(y)\right)=0
$$

which implies that $f$ is constant on $D$. Since $D$ was arbitrary if follows that $f$ must be a constant. 
We next show that $f$ is unique. We argue by contradiction. Suppose that $\Phi_{t_{n}^{\prime}} \rightarrow f^{\prime}$ over some increasing sequence $\left\{t_{n}^{\prime}\right\}$ with $t_{n}^{\prime} \uparrow \infty$ as $n \rightarrow \infty$. Without loss of generality we assume $t_{n}<t_{n}^{\prime}<t_{n+1}$ for each $n$. By (3.6) we have

$$
\Phi_{t_{n+1}}(x) \leq \mathrm{E}_{x}^{\bar{v}_{1}^{t_{n+1}}, v_{2}^{*}}\left[\Phi_{t_{n}^{\prime}}\left(X\left(t_{n+1}-t_{n}^{\prime}\right)\right)\right],
$$

and taking limits as $n \rightarrow \infty$ in (3.18) we obtain $f \leq f^{\prime}$. Reversing the roles of $f$ and $f^{\prime}$, shows that $f=f^{\prime}$.

By Lemma 3.3 we have

$$
\varphi(t, x)=\bar{\varphi}(t, x)+\int_{0}^{t} \mathrm{e}^{s-t}(\beta-\bar{\varphi}(s, 0)) \mathrm{d} s .
$$

Hence, since $\bar{\varphi}(t, x)$ converges to $\varphi^{*}(x)+f$, we obtain that $\varphi(t, x) \rightarrow \varphi^{*}(x)+\beta$ as $t \rightarrow \infty$.

\section{Risk-Sensitive Control}

In this section, we apply the results from Section 3 to study the convergence of a relative value iteration scheme for the risk-sensitive control problem which is described as follows. Let $U$ be a compact metric space and $V=\mathcal{P}(U)$ denote the space of all probability measures on $U$ with Prohorov topology. We consider the risk-sensitive control problem with state equation given by the controlled s.d.e. (in relaxed form)

$$
\mathrm{d} X(t)=b(X(t), v(t)) \mathrm{d} t+\sigma(X(t)) \mathrm{d} W(t),
$$

and payoff criterion

$$
J(x, v):=\liminf _{T \rightarrow \infty} \frac{1}{T} \ln \mathrm{E}_{x}\left[\exp \left(\int_{0}^{T} h(X(t), v(t)) \mathrm{d} t\right) \mid X(0)=x\right] .
$$

This is called the risk-sensitive payoff because in some sense it is sensitive to higher moments of the running cost and not merely its mean, thus capturing 'risk' in the sense understood in economics [13].

All processes in (4.1) are defined in a common probability space $(\Omega, \mathcal{F}, P)$ which is assumed to be complete. The process $W$ is an $\mathbb{R}^{d}$-valued standard Wiener process which is independent of the initial condition $X_{0}$ of (2.1). The control $v$ is a $V$-valued process which is jointly measurable in $(t, \omega) \in[0, \infty) \times \Omega$ and non-anticipative, i.e., for $s<t, W(t)-W(s)$ is independent of $\mathcal{F}_{s}:=$ the completion of $\sigma\left(X_{0}, v(r), W(r), r \leq s\right)$. We denote the set of all such controls (admissible controls) by $\mathcal{U}$.

Assumptions on the Data: We assume the following properties for the coefficients $b$ and $\sigma$ :

(B1) The functions $b$ and $\sigma$ are continuous and bounded, and also Lipschitz continuous in $x \in \mathbb{R}^{d}$ uniformly over $v \in V$. Also $\left(\sigma \sigma^{\top}\right)^{-1}$ is Lipschitz continuous.

(B2) For each $R>0$ there exists a constant $\kappa(R)>0$ such that

$$
z^{\top} a(x) z \geq \kappa(R)\|z\|^{2} \quad \text { for all }\|x\| \leq R \text { and } z \in \mathbb{R}^{d},
$$

where $a:=\sigma \sigma^{\top}$.

Asymptotic Flatness Hypothesis: We assume the following property: 
(B3) (i) There exists a $c>0$ and a positive definite matrix $Q$ such that for all $x, y \in \mathbb{R}^{d}$ with $x \neq y$, we have

$$
\begin{aligned}
2(b(x, v)-b(y, v))^{\top} Q(x-y)+\operatorname{tr}((\sigma(x) & \left.-\sigma(y))(\sigma(x)-\sigma(y))^{\top} Q\right) \\
& -\frac{\left\|(\sigma(x)-\sigma(y))^{\top} Q(x-y)\right\|^{2}}{(x-y)^{\top} Q(x-y)} \leq-c\|x-y\|^{2} .
\end{aligned}
$$

(ii) Let $\operatorname{Lip}(f)$ denote the Lipschitz constant of a Lipschitz continuous function $f$. Then

$$
2\left\|\sigma \sigma^{\top}\right\|_{\infty}^{2} \operatorname{Lip}(h) \operatorname{Lip}\left(\left(\sigma \sigma^{\top}\right)^{-1}\right) \leq c^{2} .
$$

The asymptotic flatness hypothesis was first introduced by 3 for the study of ergodicity in degenerate diffusions and is a little more general than the condition introduced by [7] in risk-sensitive control to facilitate the analysis of the corresponding HJB equation, which is our motivation as well. An important consequence of this condition is that if we fix a nonanticipative control process and consider two diffusion processes with this control differing only in their initial conditions, they approach each other in mean at an exponential rate 2 , Lemma 7.3.4]. This ensures a bounded gradient for the solution of the HJB equation, a key step in the analysis of its well-posedness.

We quote the following result from [6, Theorems 2.2-2.3]:

Theorem 4.1. Assume (B1)-(B3). The p.d.e.

$$
\begin{aligned}
\beta & =\min _{v \in V} \max _{w \in \mathbb{R}^{d}}\left[\tilde{L} \varphi^{*}(x, w, v)+h(x, v)-\frac{1}{2} w^{\top}\left(a^{-1}(x)\right) w\right] \\
& =\max _{w \in \mathbb{R}^{d}} \min _{v \in V}\left[\tilde{L} \varphi^{*}(x, w, v)+h(x, v)-\frac{1}{2} w^{\top}\left(a^{-1}(x)\right) w\right], \\
\varphi^{*}(0) & =0,
\end{aligned}
$$

where

$$
\tilde{L} f(x, w, v):=(b(x, v)+w) \cdot \nabla f(x)+\frac{1}{2} \operatorname{tr}\left(a(x) \nabla^{2} f(x)\right), \quad f \in C^{2}\left(\mathbb{R}^{d}\right),
$$

has a unique solution $\left(\beta, \varphi^{*}\right) \in \mathbb{R} \times C^{2}\left(\mathbb{R}^{d}\right) \cap o(\|x\|)$. Moreover, $\beta$ is the value of the risk-sensitive control problem and any measurable outer minimizing selector in (4.2) is risksensitive optimal. Also in (4.2), the supremum can be restricted to a closed ball $\tilde{V}=\overline{B_{R}}$ for

$$
R:=\frac{\operatorname{Lip}(h)}{c}+\frac{\operatorname{Lip}\left(\left(\sigma \sigma^{\top}\right)^{-1}\right) K^{2}}{2 \sqrt{c}},
$$

where $K$ is the smallest positive root (using (B3) (ii)) of

$$
\frac{\sqrt{c}}{2}\left\|\sigma \sigma^{\top}\right\|_{\infty} \operatorname{Lip}\left(\left(\sigma \sigma^{\top}\right)^{-1}\right) x^{2}-c^{5 / 4} x+\operatorname{Lip}(h)\left\|\sigma \sigma^{\top}\right\|_{\infty}=0 .
$$


For the stochastic differential game in (4.2) we consider the following relative value iteration equation:

$$
\begin{aligned}
\frac{\partial \varphi}{\partial t}(t, x) & =\min _{v \in V} \max _{w \in \tilde{V}}\left[\tilde{L} \varphi(t, x, w, v)+h(x, v)-\frac{1}{2} w^{\top}\left(a^{-1}(x)\right) w\right]-\varphi(t, 0), \\
\varphi(0, x) & =\varphi_{0}(x),
\end{aligned}
$$

where $\varphi_{0} \in C_{\mathcal{V}}\left(\mathbb{R}^{d}\right) \cap C^{2}\left(\mathbb{R}^{d}\right)$ with

$$
\mathcal{V}(x)=\frac{\left(x^{\top} Q x\right)^{1+\alpha}}{\varepsilon+\left(x^{\top} Q x\right)^{1 / 2}},
$$

for some positive constants $\varepsilon$ and $\alpha$. Here note that Assumption (B3) implies Assumption (A3) of Section 2 for the Lyapunov function $\mathcal{V}$ given above, see [2, equation (7.3.6), p. 257].

By Theorems 3.1 and 4.1 the following holds.

Theorem 4.2. Assume (B1)-(B3). For each $\varphi_{0} \in C_{\mathcal{V}}\left(\mathbb{R}^{d}\right) \cap C^{2}\left(\mathbb{R}^{d}\right), \varphi(t, x)$ converges to $\varphi^{*}(x)+\beta$ as $t \rightarrow \infty$.

The relative value iteration equation for the risk-sensitive control problem is given by

$$
\begin{aligned}
\frac{\partial \psi}{\partial t}(t, x) & =\min _{v \in V}[L \psi(t, x, v)+(h(x, v)-\ln \psi(t, 0)) \psi(t, x)] \\
\psi(0, x) & =\psi_{0}(x)
\end{aligned}
$$

where

$$
L f(x, v):=b(x, v) \cdot \nabla f(x)+\frac{1}{2} \operatorname{tr}\left(a(x) \nabla^{2} f(x)\right), \quad f \in C^{2}\left(\mathbb{R}^{d}\right) .
$$

That one has $\ln \psi(t, 0)$ instead of $\psi(t, 0)$ as the 'offset' is only natural, because we are trying to approximate the logarithmic growth rate of the cost. We have the following theorem:

Theorem 4.3. Let $\psi^{*}$ be the unique solution in the class of functions which grow no faster than $\mathrm{e}^{\|x\|^{2}}$ of the HJB equation for the risk-sensitive control problem given by

$$
\beta \psi^{*}=\min _{v \in V}\left[L \psi^{*}(x, v)+h(x, v) \psi^{*}\right], \quad \psi^{*}(0)=1 .
$$

Under assumptions (B1)-(B3) the solution $\psi(t, x)$ of the relative value iteration in (4.3) converges as $t \rightarrow \infty$ to $\mathrm{e}^{\beta} \psi^{*}(x)$ where $\beta$ is the value of the risk-sensitive control problem given in Theorem 4.1.

Proof. A straightforward calculation shows that $\psi^{*}=\mathrm{e}^{\varphi^{*}}$, where $\varphi^{*}$ is given in Theorem 4.1. Then it easily follows that $\psi(t, x)=\mathrm{e}^{\varphi(t, x)}$, where $\varphi$ is the solution of the relative value iteration for the stochastic differential game in (4.2). From Theorem 4.2, it follows that $\psi(t, x) \rightarrow \mathrm{e}^{\beta} \psi^{*}(x)$ as $t \rightarrow \infty$, which establishes the claim.

\section{Acknowledgement}

The work of Ari Arapostathis was supported in part by the Office of Naval Research under the Electric Ship Research and Development Consortium. The work of Vivek Borkar was supported in part by Grant \#11IRCCSG014 from IRCC, IIT, Mumbai. 


\section{REFERENCES}

[1] Arapostathis, A., Borkar, V.S.: A relative value iteration algorithm for nondegenerate controlled diffusions. SIAM J. Control Optim. 50(4), 1886-1902 (2012)

[2] Arapostathis, A., Borkar, V.S., Ghosh, M.K.: Ergodic control of diffusion processes, Encyclopedia of Mathematics and its Applications, vol. 143. Cambridge University Press, Cambridge (2011)

[3] Basak, G.K., Bhattacharya, R.N.: Stability in distribution for a class of singular diffusions. Ann. Probab. 20(1), 312-321 (1992)

[4] Beneš, V.E.: Existence of optimal strategies based on specified information, for a class of stochastic decision problems. SIAM J. Control 8, 179-188 (1970)

[5] Borkar, V.S., Ghosh, M.K.: Stochastic differential games: occupation measure based approach. J. Optim. Theory Appl. 73(2), 359-385 (1992)

[6] Borkar, V.S., Suresh Kumar, K.: Singular perturbations in risk-sensitive stochastic control. SIAM J. Control Optim. 48(6), 3675-3697 (2010)

[7] Fleming, W.H., McEneaney, W.M.: Risk-sensitive control on an infinite time horizon. SIAM J. Control Optim. 33(6), 1881-1915 (1995)

[8] Gilbarg, D., Trudinger, N.S.: Elliptic partial differential equations of second order, Grundlehren der Mathematischen Wissenschaften, vol. 224, second edn. Springer-Verlag, Berlin (1983)

[9] Gruber, M.: Harnack inequalities for solutions of general second order parabolic equations and estimates of their Hölder constants. Math. Z. 185(1), 23-43 (1984)

[10] Ladyženskaja, O.A., Solonnikov, V.A., Ural'ceva, N.N.: Linear and quasilinear equations of parabolic type. Translated from the Russian by S. Smith. Translations of Mathematical Monographs, Vol. 23. American Mathematical Society, Providence, R.I. (1967)

[11] Meyn, S.P., Tweedie, R.L.: Stability of Markovian processes. III. Foster-Lyapunov criteria for continuous-time processes. Adv. in Appl. Probab. 25(3), 518-548 (1993)

[12] White, D.J.: Dynamic programming, Markov chains, and the method of successive approximations. J. Math. Anal. Appl. 6, 373-376 (1963)

[13] Whittle, P.: Risk-sensitive optimal control. Wiley-Interscience Series in Systems and Optimization. John Wiley \& Sons Ltd., Chichester (1990)

Department of Electrical and Computer Engineering, The University of Texas at Austin, 1 University Station, Austin, TX 78712

E-mail address: ari@mail.utexas.edu

Department of Electrical Engineering, Indian Institute of Technology, Powai, Mumbai 400076, INDIA

E-mail address: borkar.vs@gmail.com

Department of Mathematics, Indian Institute of Technology, Powai, Mumbai 400076, India

E-mail address: suresh@math.iitb.ac.in 